Courrier du Centre international Blaise Pascal

$17 \mid 1995$

Varia

\title{
Le paradoxe pascalien
}

\section{Peter Weinmann}

\section{OpenEdition}

\section{Journals}

Édition électronique

URL : http://journals.openedition.org/ccibp/580

DOI : $10.4000 /$ ccibp. 580

ISSN : 2493-7460

\section{Éditeur}

Centre international Blaise Pascal

\section{Édition imprimée}

Date de publication : 10 décembre 1995

Pagination : 4-6

ISSN : 0249-6674

\section{Référence électronique}

Peter Weinmann, « Le paradoxe pascalien », Courrier du Centre international Blaise Pascal [En ligne],

17 | 1995, mis en ligne le 07 janvier 2016, consulté le 20 avril 2019. URL : http://

journals.openedition.org/ccibp/580 ; DOI : 10.4000/ccibp.580

Ce document a été généré automatiquement le 20 avril 2019.

Centre international Blaise Pascal 


\title{
Le paradoxe pascalien
}

\author{
Peter Weinmann
}

\section{NOTE DE L'ÉDITEUR}

Ce texte constitue l'introduction de l'article de Hugo Friedrich « Le paradoxe de Pascal : L'expression linguistique d'une forme de pensée », traduit par Peter Weinmann, et publié dans ce numéro.

1 En 1936, Hugo Friedrich publie dans la Zeitschrift für Romanische Philologie un article intitulé «Le paradoxe de Pascal», qui est le fruit d'un cours sur Pascal donné deux ans auparavant. Friedrich est alors un jeune maître de conférence (Privatdozent) qui après la publication de sa thèse sur L'Abbé Prévost en Allemagne en 1929 (sous la direction du romaniste Karl Voßler) occupe depuis 1934 son premier poste d'enseignant à l'université de Cologne.

2 Le contexte politique de l'époque a évidemment des répercussions jusqu'au sein de l'université. Celle de Cologne, fondée en 1919 par la volonté de son maire, Konrad Adenauer, jouissait d'une réputation libérale et démocratique, et malgré l'orientation catholique souhaitée par le maire, un certain pluralisme y régnait et on accordait par exemple une égalité de statut aux enseignants d'origine juive. Or, la prise de pouvoir par les nationaux-socialistes le 30 janvier 1933 change brutalement les données. Au cours de l'année 1933, tous les moyens légaux et toutes sortes de pressions sont utilisés pour chasser tous les enseignants d'origine juive ou soupçonnés de sympathie avec les communistes. Un critique du renom de Leo Spitzer, occupant la seule chaire de Romanistik à Cologne, est obligé de démissionner et part, comme tant d'autres de ses collègues juifs, en Turquie, à la jeune université d'Istanbul.

3 Si 1933 signifie pour l'Allemagne et pour le climat intellectuel dans ses universités une forte censure, la plus grande partie de la recherche publiée ne traduit pas immédiatement la nouvelle idéologie. Si une revue comme Die Neueren Sprachen, destinée à un public de professeurs de lycée, représente naturellement un enjeu politique et multiplie les déclarations d'allégeance au Führer, une revue comme la Zeitschrift für Romanische 
Philologie ne subit pas de modification visible et continue par exemple à publier régulièrement des articles de Leo Spitzer comme si de rien n'était. Ainsi, dans son essai sur Pascal, Friedrich n'hésite-t-il pas à déclarer sa dette envers le « juif » Ernst Cassirer.

Certes, l'intention immédiate du texte n'est pas de faire de la résistance idéologique. En choisissant de faire l'apologie d'un auteur chrétien, Friedrich situe le débat sur un autre terrain. La Grande Guerre qui a opposé la France et l'Allemagne hantait encore les esprits et avait conforté l'idée d'un antagonisme irréductible et «naturel » entre les deux pays, entre «civilisation" française (interprétée du côté allemand comme "rationnelle, superficielle, décadente, féminine » etc.) et «Kultur » allemande (à laquelle on réservait les épithètes " géniale, profonde, héroïque, virile » etc.). D’un côté comme de l'autre, les clichés étaient soigneusement développés, même, ou plutôt surtout, par les spécialistes en la matière. La Romanistik allemande, mais aussi les germanistes français débattaient avec passion des recherches récentes de la Völkerpsychologie, la psychologie des peuples, qui tentait d'expliquer les différences des esprits et des mentalités comme une donnée naturelle, constante à travers les siècles ${ }^{1}$.

En même temps, de plus en plus de voix s'élevaient pour tirer les conséquences de cette «incompatibilité » culturelle. Ainsi lit-on en 1934 dans la revue Die Neueren Sprachen: «Plus nous pénétrons dans l'histoire des rapports intellectuels de ces deux peuples, plus nous avons l'impression que nous restons étrangers l'un à l'autre [...], malgré les études mutuelles. » L'objectif avoué de l'apprentissage du français dans les écoles ne pouvait être alors que «faire comprendre à nos élèves la nature allemande au miroir du caractère national étranger ${ }^{2} »$.

6 Pour définir l'«essence de la France ", une référence obligatoire était la littérature classique du XVII ${ }^{\text {e }}$ siècle, incarnation de l'esprit français par excellence : sens de l'ordre, mesure, rationalité, méthode etc. en étaient les mots-clefs.

$7 \quad$ Il va de soi que cette image stéréotypée était largement le résultat de choix idéologiques et empêchait parfois un regard objectif sur les auteurs classiques en question. Dans le compte rendu d'un livre sur la langue française ${ }^{3}$, Friedrich met en garde contre toute généralisation hâtive d'une Völkerpsychologie linguistique qui oublierait, devant le «rationalisme» prétendument omniprésent de langue française, que c'est dans cette langue que s'exprime la profondeur de Pascal, le tempérament irrationnel de Diderot ou la musicalité de Verlaine. De même dans son article sur Pascal, Friedrich indique discrètement, avec en exergue une citation de Novalis, que l'opposition entre classicisme français et romantisme allemand n'est pas forcément insurmontable.

Pour ce qui est de Pascal, l'article de Friedrich s'insurge à la fois contre deux simplifications: ranger Pascal du côté de la théologie, ou bien le ranger simplement parmi les rationalistes. Friedrich s'efforcera d'une part de montrer la portée humaine et universelle de la réflexion pascalienne qui s'adresse avant tout au non-spécialiste, croyant ou incroyant. D'autre part, il montrera comment Pascal utilise effectivement les outils rationnels tirés de la géométrie pour décrire la nature humaine, mais pour mieux montrer ensuite les limites de la raison. Pour « définir l'indéfinissable», Pascal se sert d'un instrument qu'il manie plus habilement que ses contemporains : le paradoxe, qui est, selon Friedrich, le grand principe de base de la pensée pascalienne, " une formule si caractéristique des Pensées, dont tout le texte des Pensées est entremêlé avec un esprit de système presque mécanique et qui détermine la langue pascalienne jusque dans ses dernières ramifications $s^{4}$ ». Le paradoxe est lui-même de nature paradoxale : il permet de décrire clairement, dans des termes rationnels et parfaitement compréhensibles, mais il 
décrit un état de choses contradictoire, qui n'est pas accessible à la raison, qui ne peut pas l'être, l'homme étant « un monstre incompréhensible ». Par le paradoxe, la raison nous conduit aux limites de ses possibilités, suggérant un au-delà qu'elle ne saurait atteindre.

Quatorze années plus tard, en 1950, Hugo Friedrich consacre un deuxième article à Pascal, plus exactement la transcription d'une conférence qui retrace brièvement quelques thèmes majeurs des Pensées. Après les horreurs de la Deuxième Guerre Mondiale, la leçon de Pascal dut revêtir alors une vérité poignante pour l'auditoire et l'orateur qui pouvait se dispenser de souligner l'actualité renouvelée du paradoxe pascalien: «Ce qui est troublant avec cette créature ambiguë qu'est l'homme, c'est qu'elle consiste en misère et grandeur, en impuissance et majesté, disharmonieuse, contradictoire, désunie [...]. Et c'est cela qui est spécifique et incomparable dans Pascal, qu'il médite sur cette dualité troublante et qu'il trouve dans les états les plus simples de la condition humaine le caractère paradoxal d'une nature prédestinée aussi bien à la grandeur qu'à la déchéance. ${ }^{5}$ $\gg$

10 La traduction qui suit voudrait apporter au lecteur non-germanophone le témoignage d'une lecture de Pascal, certes vieille de soixante ans, mais encore capable, je le souhaiterais, d'enrichir l'exégèse pascalienne de nos jours.

\section{NOTES}

1. Une synthèse sur ce sujet par Michael Nerlich a été publiée récemment dans la revue Lendemains 69/70, 1993.

2. Erwin Güntsch, «Die Zuspitzung der geistigen Auseinandersetzung mit Frankreich », Die Neueren Sprachen 42, 1934, p. 298-307; ici: p. 306.

3. H. Friedrich, compte rendu de: W. von Wartburg, Évolution et Structure de la langue française, Leipzig-Berlin, Teubner, 1934, dans : Zeitschrift für Romanische Philologie LVI, 1936, p. 472.

4. Page 4 de la présente traduction.

5. Hugo Friedrich, «Pascal. Ein Vortrag », Deutsche Vierteljahrsschrift für Literaturwissenschaft und Geistesgeschichte 24, 1950, p. 287-303 ; ici p. 301.

\section{INDEX}

Mots-clés : Friedrich (Hugo), Pascal, paradoxe

Keywords : Friedrich (Hugo), Pascal, paradox 